\title{
Localization of light: Dual symmetry between absorption and amplification
}

\author{
J. C. J. Paasschens \\ Instituut-Lorentz, University of Leiden, P.O. Box 9506, 2300 RA Leiden, The Netherlands \\ and Philips Research Laboratories, 5656 AA Eindhoven, The Netherlands \\ T. Sh. Misirpashaev \\ Instituut-Lorentz, University of Leiden, P.O. Box 9506, 2300 RA Leiden, The Netherlands \\ and Landau Institute for Theoretical Physics, 2 Kosygin Street, Moscow 117334, Russia \\ C. W. J. Beenakker \\ Instituut-Lorentz, University of Leiden, P.O. Box 9506, 2300 RA Leiden, The Netherlands
}

(Received 28 May 1996)

\begin{abstract}
We study the propagation of radiation through a disordered waveguide with a complex dielectric constant $\varepsilon$, and show that dual systems, which differ only in the sign of the imaginary part of $\varepsilon$, have the same localization length. Paradoxically, absorption and stimulated emission of radiation suppress the transmittance of the waveguide in the same way. [S0163-1829(96)07338-9]
\end{abstract}

Localization of electromagnetic waves in a random medium has attracted much interest, ${ }^{1}$ since the original proposals of $\mathrm{John}^{2}$ and Anderson. ${ }^{3}$ An essential difference with localization of electrons is the absence of a conservation law for photons. Light is absorbed or amplified-while retaining the phase coherence-if the dielectric constant has a nonzero imaginary part. The intensity of the radiation which has propagated without reflection over a distance $L$ is then multiplied by a factor $e^{\sigma L}$, with $\sigma$ negative (positive) for absorption (amplification). The interplay of absorption and localization has been studied extensively. ${ }^{2-8}$ For the onedimensional problem of a disordered single-mode waveguide (length $L$, mean free path $l$ ), the result for the transmittance $T$ (being the ratio of transmitted and incident flux) is ${ }^{6,7}$

$$
\langle\ln T\rangle=\left(\sigma-l^{-1}\right) L,
$$

where $\langle\cdots\rangle$ denotes an average over disorder. Equation (1) was derived for $\sigma<0$, corresponding to absorption.

In this paper we address the question: What happens for amplification? Since $T=e^{\sigma L}$ in the absence of reflection for both positive and negative $\sigma$, one might surmise that Eq. (1) holds both for absorption and amplification. This is correct for short waveguides. However, as first noted by Zhang, ${ }^{9}$ the asymptotic result for $L \rightarrow \infty$ is

$$
\langle\ln T\rangle=\left(-|\sigma|-l^{-1}\right) L+\mathcal{O}(1) .
$$

We will show that exponential decay of the transmittance in the case of amplification, $\langle\ln T\rangle \simeq-L / \xi$, is in fact implied by its exponential decay in the case of absorption, with a duality relation between decay lengths:

$$
\xi(\sigma)=\xi(-\sigma) .
$$

This duality relation extends beyond the strictly onedimensional case of Eq. (2), the only essential ingredient being an exponentially decaying transmittance in an absorb- ing system. Contrary to intuition, amplification suppresses the transmittance in the large- $L$ limit just as much as absorption does.

Experimentally, a random amplifying medium can be realized in a turbid laser dye or a powdered laser crystal. ${ }^{10-12}$ Stimulated emission of radiation leads to a dielectric constant with a negative imaginary part, corresponding to $\sigma>0$. We do not present a complete theory for such a "random laser," because we ignore spontaneous emission. ${ }^{13}$ Still, because of the different time scales for stimulated and spontaneous emissions, we believe that a time-resolved experiment in a wave guide geometry might give evidence for the localization of stimulated emission, before spontaneous emission sets in.

To prove the duality relation (3) we consider the propagation of monochromatic radiation (scalar amplitude $E$, wave number $k$ ), described by the Helmholtz equation

$$
\mathcal{H} E(\vec{r})=0, \quad \mathcal{H}=\nabla^{2}+k^{2} \varepsilon(\vec{r})
$$

(We suppose that all polarization-sensitive phenomena are absent.) Disorder leads to spatial fluctuations of the real part $\varepsilon^{\prime}$ of the dielectric constant. In the absence of disorder $\varepsilon^{\prime}=1$. A nonzero imaginary part $\varepsilon^{\prime \prime}$ makes the system nonconservative. For the general duality relation it is irrelevant whether $\varepsilon^{\prime \prime}$ depends on $\vec{r}$ or not. The sign of $\varepsilon^{\prime \prime}$ determines whether the system is locally absorbing $\left(\varepsilon^{\prime \prime}>0\right)$ or amplifying $\left(\varepsilon^{\prime \prime}<0\right)$. For a constant $\varepsilon^{\prime \prime}$ the parameter $\sigma$ introduced above is given by

$$
\sigma=-2 k \operatorname{Im} \sqrt{1+i \varepsilon^{\prime \prime}},
$$

where the argument of the square root is chosen in the interval $(-\pi / 2, \pi / 2)$.

The dual symmetry underlying Eq. (3) is formulated in its general form in terms of scattering matrices. We assume that the system consists of a scattering region of length $L$, in which $\varepsilon=\varepsilon^{\prime}+i \varepsilon^{\prime \prime}$, embedded in an $N$-mode waveguide with 


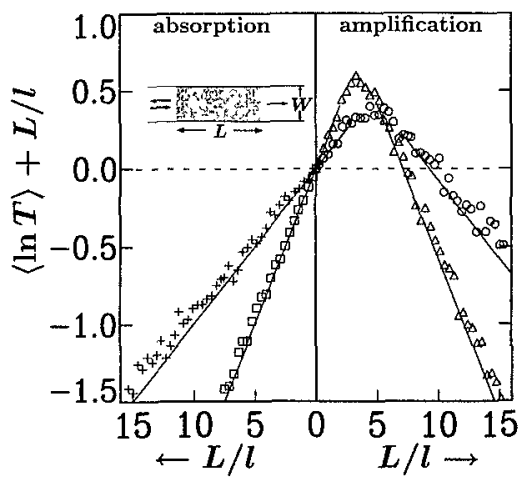

FIG. 1. Numerical simulation of the transmittance of a singlemode waveguide $\left(W=d, k^{2}=0.5 d^{-2}, \delta \varepsilon=0.2, l=521 d, N=1\right)$, averaged over $10^{4}$ realizations of the disorder. The right half of the figure is for amplification (circles: $\gamma=0.1$; triangles: $\gamma=0.2$ ), the left half is for absorption (crosses: $\gamma=-0.1$; squares: $\gamma=-0.2$ ). The solid lines are the analytical asymptotes from Eq. (15). Their slope is independent of the sign of $\gamma$, in agreement with the duality relation (3). The inset shows the geometry considered.

$\varepsilon=1$ (see Fig. 1, inset). The scattering matrix $S$ is a $2 N \times 2 N$ matrix relating incoming and outgoing modes. It has the block structure

$$
S=\left(\begin{array}{l}
r t^{\prime} \\
t r^{\prime}
\end{array}\right),
$$

where $r, r^{\prime}$ are the reflection matrices and $t, t^{\prime}$ the transmission matrices. The transmittances and reflectances are defined as

$$
\begin{array}{cc}
T=N^{-1} \operatorname{Tr} t t^{\dagger}, & R=N^{-1} \operatorname{Tr} r r^{\dagger}, \\
T^{\prime}=N^{-1} \operatorname{Tr} t^{\prime} t^{\prime \dagger}, & R^{\prime}=N^{-1} \operatorname{Tr} r^{\prime} r^{\prime \dagger} .
\end{array}
$$

Here $T$ and $R$ are the transmitted and reflected flux divided by the incident flux from the left. Similarly, $T^{\prime}$ and $R^{\prime}$ correspond to incident flux from the right. By taking the trace in Eq. (7) we are assuming diffuse illumination, i.e. that the incident flux is equally distributed over the $N$ modes. In the absence of gain or loss $\left(\varepsilon^{\prime \prime}=0\right)$ the scattering matrix is unitary, $S S^{\dagger}=1$. This relation expresses flux conservation and relies upon Hermiticity of the Helmholtz operator, $\mathcal{H}=\mathcal{H}^{\dagger}$ at $\varepsilon^{\prime \prime}=0$. For nonzero $\varepsilon^{\prime \prime}$ we have $\mathcal{H}\left(-\varepsilon^{\prime \prime}\right)=\mathcal{H}^{\dagger}\left(\varepsilon^{\prime \prime}\right)$, which implies the duality relation

$$
S\left(-\varepsilon^{\prime \prime}\right) S^{\dagger}\left(\varepsilon^{\prime \prime}\right)=1
$$

[If $\varepsilon^{\prime \prime}$ depends on $\vec{r}$ the duality relation refers to a change of sign for the complete function $\left.\varepsilon^{\prime \prime}(\vec{r}) \rightarrow-\varepsilon^{\prime \prime}(\vec{r}).\right]$

Let us now examine the consequences of the duality relation (8) for the reflection and transmission matrices of two systems which differ only in the sign of $\varepsilon^{\prime \prime}$. (We call these systems "dual.") We assume that one of the two dual systems (indicated by a subscript -) is globally absorbing, so that all elements of $t_{-}$and $t_{-}^{\prime}$ tend to zero in the limit $L \rightarrow \infty$, while $r_{-}$and $r_{-}^{\prime}$ remain finite. Expanding the inverse of $S_{-}$to first order in the transmission matrices and equating the result to $S_{+}^{\dagger}$ we find

$$
\begin{gathered}
r_{+}^{\dagger}=r_{-}^{-1}+\mathcal{O}\left(t^{2}\right), \quad r_{+}^{\prime \dagger}=r_{-}^{\prime-1}+\mathcal{O}\left(t^{2}\right), \\
t_{+}^{\dagger}=-r_{-}^{-1} t_{-}^{\prime} r_{-}^{\prime-1}+\mathcal{O}\left(t^{2}\right) .
\end{gathered}
$$

We introduce the transmission and reffection eigenvalues $\mathcal{T}_{n}, \mathcal{T}_{n}^{\prime} \mathcal{R}_{n}, \mathcal{R}_{n}^{\prime}$ being the eigenvalues of, respectively, $\mathbf{T}=t t^{\dagger}, \mathbf{T}^{\prime}=t^{\prime} t^{\prime \dagger}, \mathbf{R}=r r^{\dagger}, \mathbf{R}^{\prime}=r^{\prime} r^{\prime \dagger}$. Because of timereversal symmetry $S_{-}^{*} S_{+}^{*}=1$. Together with Eq. (8) this implies that $S$ is a symmetric matrix. It follows that $t^{\prime}=t^{\mathrm{T}}$, hence $\mathcal{T}_{n}=\mathcal{T}_{n}^{\prime}$ and $T=T^{\prime}$. The reflectances $R$ and $R^{\prime}$ may differ. Equation (9) directly yields a duality relation for the reflection eigenvalues in the limit $L \rightarrow \infty$,

$$
\mathcal{R}_{n}\left(\varepsilon^{\prime \prime}\right)=\mathcal{R}_{n}^{-1}\left(-\varepsilon^{\prime \prime}\right)
$$

Equations (9) and (10) together imply that the matrices $\mathbf{T}_{-}^{\prime} \mathbf{R}_{-}^{-1}$ and $\mathbf{T}_{+} \mathbf{R}_{+}^{\prime-1}$ have the same eigenvalues. The duality relation for the transmission eigenvalues follows from the following lemma:

Let $A(L)$ be a matrix function of $L$ with exponentially decreasing eigenvalues $a_{n}(L)$. The eigenvalue localization lengths $\xi_{n}$ are defined by $\xi_{n}^{-1}=-\lim _{L \rightarrow \infty} L^{-1} \ln a_{n}(L)$. Let $B(L)$ be another nonsingular matrix function whose elements remain finite as $L \rightarrow \infty$. Then the matrix $A B$ has the same eigenvalue localization lengths as $A$.

It follows that the matrices $\mathbf{T}_{+}, \mathbf{T}_{+} \mathbf{R}_{+}^{\prime}{ }^{-1}, \mathbf{T}_{-}^{\prime} \mathbf{R}_{-}^{-1} \mathbf{T}_{-}^{\prime}$, and hence $\mathbf{T}_{-}$all have the same eigenvalue localization lengths. Explicitly,

$$
-\lim _{L \rightarrow \infty} L^{-1} \ln \mathcal{T}_{n}\left(\varepsilon^{\prime \prime}\right)=-\lim _{L \rightarrow \infty} L^{-1} \ln \mathcal{T}_{n}\left(-\varepsilon^{\prime \prime}\right)
$$

The transmittance $T=N^{-1} \Sigma_{n} \mathcal{T}_{n}$ is dominated by the largest transmission eigenvalue, which is the $\mathcal{T}_{n}$ with the largest localization length: $\xi=\max \left(\xi_{1}, \xi_{2}, \ldots, \xi_{N}\right)$. This completes the proof of Eq. (3), since we have shown that all, and in particular the largest, transmission eigenvalues of dual systems have the same localization length.

The case $N=1$ of a single-mode waveguide can be analyzed in more detail. The joint probability distribution $P(R, T, L)$ of reflectance and transmittance evolves with increasing $L$ according to a Fokker-Planck equation,

$$
\begin{aligned}
l \frac{\partial P}{\partial L}= & -\frac{\partial}{\partial R}\left[(1-R)^{2}+2 \sigma l R\right] P+\frac{\partial^{2}}{\partial R^{2}} R(1-R)^{2} P \\
& -\frac{\partial}{\partial T} T(\sigma l-1+R) P+\frac{\partial^{2}}{\partial T^{2}} T^{2} R P \\
& -2 \frac{\partial^{2}}{\partial R \partial T} T R(1-R) P .
\end{aligned}
$$

The parameters $l$ and $\sigma$ are spatial averages over length scales much smaller than the total length $L$ of the waveguide. Equation (13) holds if the wavelength $\lambda$ is much smaller than both $l$ and $1 /|\sigma|$. (This is not a restrictive assumption for an optical system.) For $\sigma<0$ (absorption), this equation is equivalent to the moment equation of Freilikher, Pustilnik, and Yurkevich. ${ }^{7}$ For $\sigma>0$ (amplification) their method of moments cannot be used, because all moments of $R$ diverge when $L$ exceeds the laser threshold $L_{c} \cdot{ }^{14}$ The derivation of 


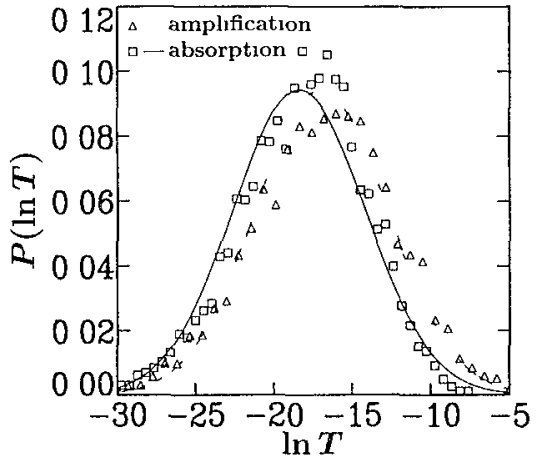

FIG 2 Probability distribution of the logarithm of the transmittance of a single mode wavegurde, for $L / l=154$ and $\gamma=02$ (tri angles, dashed curve), $\gamma=-02$ (squares, solid curve) The data points are provided by a numerical simulation (same parameters as in Fig 1), the curves are a Gaussian distribution of $\ln T$ with mean and variance given by Eqs (15) and (16) There is a slight offset between the distributions for absorption and amplification because the system is not fully in the large- $L$ limit

$\mathrm{Eq}$ (13) proceeds along the lines of Ref 15, where the case $\sigma=0$ was considered On integration over $T$ it reduces to a well known ${ }^{16-18}$ Fokker-Planck equation for $P(R, L)$ $=\int d \operatorname{TP}(R, T, L)$ The limit $L \rightarrow \infty$ of $P(R, L)$ was studied in Refs 14,17 , and 18 In terms of the variable $\mu=1 /(R-1)$ it reads

$$
P(\mu)= \begin{cases}2 \gamma e^{-2 \gamma \mu} \theta(\mu), & \text { for } \gamma>0, \\ -2 \gamma e^{-2 \gamma(1+\mu)} \theta(-1-\mu), & \text { for } \gamma<0,\end{cases}
$$

where we have defined $\gamma=\sigma l$ The function $\theta(x)=1$ for $x>0$ and 0 otherwise

Using this asymptotic distribution we have computed from $\mathrm{Eq}$ (13) the first two moments of $\ln T$ in the large- $L$ limit The result for the average is

$$
\begin{gathered}
\langle\ln T\rangle=-(1+|\gamma|) L / l+2 c(\gamma), \\
c(\gamma)= \begin{cases}0, & \text { for } \gamma<0, \\
\mathrm{C}+\ln 2 \gamma-e^{2 \gamma} \operatorname{El}(-2 \gamma), & \text { for } \gamma>0,\end{cases}
\end{gathered}
$$

where $\mathrm{C}$ 1s Euler's constant and $\mathrm{E}_{1}(x)=\int_{-\infty}^{x} d t e^{t} / t$ is the exponential integral [ $c(\gamma) \approx-2 \gamma \ln \gamma$ if $0<\gamma \ll 1]$ Equation (15) agrees with Refs 6,7 , and 9, and demonstrates that the 1nverse localization length $\xi^{-1}(\sigma)=(1+|\gamma|) l^{-1}=l^{-1}+|\sigma|$ is independent of the sign of $\sigma$-in accordance with the general duality relation (3) The result for the variance is

$$
\text { var } \ln T=2\left[1+2|\gamma| e^{2|\gamma|} \operatorname{Er}(-2|\gamma|)\right] L / l+\mathcal{O}(1)
$$

in agreement with Ref 7 for $y<0$ Note that $\sqrt{\operatorname{var} \ln T} \ll\langle\ln T\rangle$ for $L / l \gg 1$ Evaluation of higher moments shows that the distribution of $\ln T$ tends to a Gaussian for $L \rightarrow \infty$ (The tails are non-Gaussian, but contain negligible weight )

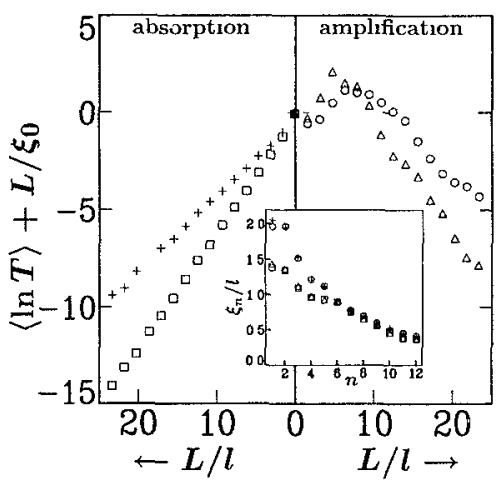

FIG 3 Numerical simulation of the transmittance of a mult mode waveguide $\left(W=25 d, k^{2}=20 d^{-2}, \delta \varepsilon=0375, l=296 d\right.$ $N=12$ ), averaged over 50 realizations of the disorder The parameter $\xi_{0}=(N+1) l / 2$ is the localization length of the system in the absence of absorption or amplification The right half of the figure is for amplification (circles $\sigma=00035 d^{-1}$, triangles $\sigma=00071 d^{1}$ ), the left half is for absorption (crosses $\sigma=-00035 d^{\text {l }}$, squares $\sigma=-00071 d^{-1}$ ) The inset shows the eigenvalue localization lengths, $\xi_{n}^{-1} \equiv-\lim _{L \rightarrow \infty} L^{-1} \ln \mathcal{T}_{n} \quad$ These lengths $\xi_{n}$ were computed from the $L$ dependence of $T_{n}$ for $L$ up to $40 l$ and a single realization of the disorder The duality between absorption and amplification is verified with good accuracy

These results hold in the laige- $L$ limit For short waveguides instead of Eq $(15)$ one has $\langle\ln T\rangle=-(1-\gamma) L / l$ The crossover length is the lasing treshold $L_{c} \simeq l c(\gamma) /|\gamma|$, at which $\langle T\rangle$ diverges for $\gamma>0$ Below this length stimulated emission enhances transmission through the waveguide On larger length scales stimulated emiscion reduces transmission In contrast, the reflectance is enhanced on every length scale 141718

To test these analytical predictions for $N=1$, and to in vestigate also the multimode case, we have numerically solved a discretized version of the Helmholtz equation (4), on a two-dimensional square lattice (lattice constant $d$, length $L$, width $W$ ) The real part $\varepsilon^{\prime}$ of the dielectric con stant was chosen randomly from site to site with a uniform distribution between $1 \pm \delta \varepsilon$ The imaginary part $\varepsilon^{\prime \prime}$ of the dielectric constant had the same value at each site The scattering matrix for the multımode case was computed using the recursive Green function technique, originally developed for the electronic Anderson model ${ }^{19}$ (For the case $N=1$ a transfer-matrix method ${ }^{89}$ turned out to be more convenient ) Simulations with $\varepsilon^{\prime \prime}=0$ were used to obtain $l$, from the relation $^{20}$

$$
-\lim _{L \rightarrow \infty} L^{-1}\langle\ln T\rangle=\left[\frac{1}{2}(N+1) l\right]^{l}
$$

The parameter $\sigma$ was determined from Eq (5) Results for the single-mode case are shown in Figs 1 and 2 and for the multimode case in Fig 3 The duality relation between the localization lengths for absorption and amplitication is vell fied with good accuracy, both for the single-mode and tor the multımode case Furthermore, for $N=1$ we find good dgitc ment with the results (15)-(16) of the Fokker Planck equa tion 
In conclusion, we have demonstrated that stimulated emission of radiation in a disordered waveguide reduces the decay length, in the same way as absorption does. This paradoxical result is an immediate consequence of the exact duality relation (8) between the scattering matrices of two systems with complex conjugated dielectric constants. The dual symmetry between absorption and amplification has been supported by an explicit computation of the decay lengths, both analytically (for the single-mode case) and numerically (for the single- and multimode cases).

We acknowledge useful discussions with P. W. Brouwer and K. M. Frahm. This work was supported by the Dutch Science Foundation NWO/FOM.
${ }^{1}$ S. John, Phys. Today 44 (5), 32 (1991); Scattering and Localization of Classical Waves in Random Media, edited by P. Sheng (World Scientific, Singapore, 1990).

${ }^{2}$ S. John, Phys. Rev. Lett. 53, 2169 (1984).

${ }^{3}$ P. W. Anderson, Philos. Mag. B 52, 505 (1985).

${ }^{4}$ R. L. Weaver, Phys. Rev. B 47, 1077 (1993).

${ }^{5}$ M. Yosefin, Europhys. Lett. 25, 675 (1994).

${ }^{6}$ R. Rammal and B. Doucot, J. Phys. (France) 48, 509 (1987).

${ }^{7}$ V. Freilikher, M. Pustilnik, and I. Yurkevich, Phys. Rev. Lett. 73, 810 (1994).

${ }^{8}$ A. K. Gupta and A. M. Jayannavar, Phys. Rev. B 52, 4156 (1995).

${ }^{9}$ Z. Q. Zhang, Phys. Rev. B 52, 7960 (1995).

${ }^{10}$ N. M. Lawandy, R. M. Balachandran, A. S. L. Gomes, and E. Sauvain, Nature 368, 436 (1994).

${ }^{11}$ W. L. Sha, C.-H. Liu, and R. R. Alfano, Opt. Lett. 19, 1922 (1994).
${ }^{12}$ D. S. Wiersma, M. P. van Albada, and A. Lagendijk, Phys. Rev. Lett. 75, 1739 (1995)

${ }^{13}$ A. Yu. Zyuzin, Phys. Rev. E 51, 5274 (1995).

${ }^{14}$ C. W. J. Beenakker, J. C. J. Paasschens, and P. W. Brouwer, Phys. Rev. Lett. 76, 1368 (1996).

${ }^{15}$ P. A. Mello, P. Pereyra, and N. Kumar, Ann. Phys. (N.Y.) 181, 290 (1988).

${ }^{16} \mathrm{M}$. E. Gertsenshtein and V. B. Vasil'ev, Teor. Veroyatn. Primen. 4, 424 (1959); 5, 3(E) (1960) [Theor. Probab. Appl. 4, 391 (1959); 5, 340(E) (1960)].

${ }^{17}$ W. Kohler and G. C. Papanicolaou, SIAM J. Appl. Math. 30, 263 (1976).

${ }^{18}$ P. Pradhan and N. Kumar, Phys. Rev. B 50, 9644 (1994).

${ }^{19}$ H. U. Baranger, D. P. DiVincenzo, R. A. Jalabert, and A. D. Stone, Phys. Rev. B 44, 10637 (1991).

${ }^{20}$ O. N. Dorokhov, Solid State Commun. 51, 381 (1984). 